\title{
Clinical Study \\ Impact of Cardiac Computed Tomographic Angiography Findings on Planning of Cancer Therapy in Patients with Concomitant Structural Heart Disease
}

\author{
Iyad N. Daher, Jose Banchs, Syed Wamique Yusuf, Elie Mouhayar, \\ Jean-Bernard Durand, and Gregory Gladish
}

The University of Texas MD Anderson Cancer Center, Houston, TX 77030, USA

Correspondence should be addressed to Iyad N. Daher, indaher@mdanderson.org

Received 22 September 2010; Accepted 26 January 2011

Academic Editor: Daniel J. Lenihan

Copyright (c) 2011 Iyad N. Daher et al. This is an open access article distributed under the Creative Commons Attribution License, which permits unrestricted use, distribution, and reproduction in any medium, provided the original work is properly cited.

\begin{abstract}
Background. Exclusion of underlying coronary artery disease (CAD) is essential in the diagnosis of chemotherapy-induced cardiomyopathy. Presence and severity of CAD can also impact the choice of therapy in cancer patients. The value of cardiac computed tomographic angiography (CCTA) in this setting has not been reported. Methods. We collected data on the clinical presentation and indications for CCTA performed from January to December 2008 at the University of Texas MD Anderson Cancer Center (MDACC). All examinations were performed using a 64-detector scanner. CCTA results and subsequent treatment decisions were examined. Results. A total of 80 patients underwent CCTA during the study period for the following indications (not mutually exclusive): cardiomyopathy of unknown etiology in 33 pts (41.3\%), chest pain in 32 (40.0\%), abnormal stress test in $16(20.0 \%)$, abnormal cardiac markers in $8(10.0 \%)$, suspected cardiac mass or thrombus in 7 (8.8\%). Chemotherapy-induced cardiomyopathy was diagnosed in 18 pts $(22.5 \%)$. Severe CAD was detected in 22 pts $(27.5 \%)$; due to concomitant advanced cancer or patient refusal, only 12 underwent coronary angiogram. Of these, 4 pts (5\% of total) underwent coronary artery bypass grafting. A total of 41 pts $(51.3 \%)$ had their cancer management altered based on CCTA findings. Conclusion. CCTA is useful in evaluating cancer pts with structural heart disease and can have an impact on the management of cancer and cardiac disease.
\end{abstract}

\section{Introduction}

Suspected coronary artery disease (CAD) in patients with a concurrent malignancy greatly impacts prognosis and treatment decisions. Faced with both diseases, appropriate prioritization of care is needed, as some cancer treatments may be cardiotoxic [1] or lead to blood dyscrasias that could discourage the use of commonly prescribed cardiac medications, such as aspirin or heparin products. On the other hand, planning of cardiac invasive testing requires clinicians to take into account cancer status and possible delays in care. Noninvasive coronary anatomical imaging could therefore help with accurate treatment planning prior to using invasive strategies.

Exclusion of significant obstructive CAD in patients with left ventricular (LV) dysfunction and a prior history of exposure to cardiotoxic chemotherapy agents are both needed for the diagnosis of chemotherapy-induced cardiomyopathy (CIC) [2]. In addition, some cancer patients presenting with other findings suggestive of structural heart disease may need an accurate anatomical coronary evaluation. While invasive coronary angiography (ICA) remains the procedure of choice in patients with high pretest probability of CAD, it is not always justified in those with low to intermediate suspicion of CAD [3]. It also carries a risk of bleeding or infectious complications, which may be more likely in cancer patients because of blood dyscrasias or immunosuppression. Ongoing chemotherapy or radiation treatment may also further delay ICA. There is therefore a need for an accurate noninvasive modality to define the coronary anatomy in some cancer patients. Cardiac computed tomographic angiography (CCTA) is such a modality, and has been shown to correlate well with ICA [4-6]. Specifically, its negative predictive value in excluding $\mathrm{CAD}$ in the presence 
TABLE 1: Baseline cardiac CT angiogram population characteristics.

\begin{tabular}{lc}
\hline \multicolumn{2}{c}{ Population characteristics } \\
\hline Age in years (mean $\pm \mathrm{SD}^{*}$ ) & $58.8 \pm 12.8$ \\
Male gender (\%) & $45(56.3)$ \\
Known coronary artery disease (\%) & $7(8.8)$ \\
Hypertension (\%) & $38(47.5)$ \\
Hyperlipidemia (\%) & $29(36.3)$ \\
Diabetes mellitus (\%) & $10(12.5)$ \\
Smoking (\%) & $5(6.3)$ \\
Previous chest/mediastinal radiation (\%) & $11(13.8)$ \\
Hematologic malignancy (\%) & $36(45.0)$ \\
\hline
\end{tabular}

* SD: standard deviation.

TABLE 2: Indications for cardiac computed tomographic angiography.

\begin{tabular}{lc}
\hline CCTA* indications $^{*}$ & $N(\%)$ \\
\hline Left ventricular dysfunction & $33(41.3)$ \\
Chest pain/dyspnea & $32(40.0)$ \\
Abnormal stress test & $16(20.0)$ \\
Abnormal cardiac biomarkers & $8(10.0)$ \\
Miscellaneous $^{\dagger}$ & $13(16.3)$ \\
\hline${ }^{*}$ CCTA: cardiac computed tomographic angiography. \\
${ }^{\dagger}$ Miscellaneous indications included suspected tumor and/or thrombus (7 \\
patients) and suspected pulmonary embolism (6 patients).
\end{tabular}

of a normal scan is very high [7]. We sought to investigate the impact of CCTA findings on cancer treatment decisions.

\section{Methods}

This retrospective data review study was approved by the Institutional Review Board (IRB). The clinical presentation and baseline characteristics of patients who underwent CCTA between January and December 2008 at the University of Texas MD Anderson Cancer Center (MDACC) were reviewed. The indications for CCTA were collected, as well as contraindications to invasive angiography, when explicitly documented. The downstream impact of CCTA results on patient care decision-making was ascertained for both cardiac and cancer treatment by review of the medical records. Specific diagnostic or therapeutic steps were considered to be based on CTA results only when medical records explicitly referenced the CTA results and directly linked those decisions with these findings. All statistical analysis was performed using NCSS2001 (Kaysville, Utah).

Prior to CCTA, patients with heart rates above 65 beats per minute on breath holding received beta-blockers (metoprolol, up to $100 \mathrm{mg}$ orally and/or up to $20 \mathrm{mg}$ intravenously). Patients needing heart rate control, but with contraindications to beta-blockers were managed with oral calcium-channel blockers. When blood pressure allowed, patients were also given sublingual nitroglycerin spray $0.4 \mathrm{mg}$ immediately prior to undergoing scan. All studies were performed using a 64-detector scanner (Lightspeed VCT,
TABLE 3: Relative contraindications for invasive coronary angiography.

\begin{tabular}{lc}
\hline Invasive angiography contraindications & $N(\%)$ \\
\hline Low to moderate probability for CAD* & $49(61.3)$ \\
Leucopenia/thrombocytopenia & $15(19.2)$ \\
Concomitant chemotherapy or radiation & $20(25.0)$ \\
$\geq 1$ above contraindication & $72(90)$ \\
\hline *CAD: coronary artery disease. & \\
Contraindications are not mutually exclusive.
\end{tabular}

TABLE 4: Coronary findings of cardiac computed tomographic angiography.

\begin{tabular}{|c|c|}
\hline CCTA* results & $N(\%)$ \\
\hline Normal coronary arteries & $21(26.3)$ \\
\hline Mild to moderate $\mathrm{CAD}^{\dagger}$ & $37(46.2)$ \\
\hline Severe $^{\ddagger}$ CAD & $22(27.5)$ \\
\hline
\end{tabular}

General Electric Healthcare (GEHC), Milwaukee, WI). A calcium scoring scan was performed using $2.5 \mathrm{~mm}$ collimation and $25 \mathrm{~cm}$ display field of view with prospective cardiac triggering without intravenous contrast material. $150 \mathrm{~mL}$ of $320 \mathrm{mgI} / \mathrm{mL}$ contrast material (iodixanol, Visipaque 320, GEHC) was administered at $5 \mathrm{~mL} / \mathrm{sec}$, followed by $50 \mathrm{~mL}$ of normal saline at $5 \mathrm{~mL} / \mathrm{sec}$. A fixed scan delay of 30 seconds was used. Cardiac scanning was performed with $0.625 \mathrm{~mm}$ collimation using retrospective cardiac gating and electrocardiographic (ECG) dose modulation. In patients with significant beat to beat heart rate variability or frequent ectopic beats, ECG dose modulation was not used. Data was reconstructed at $0.625 \mathrm{~mm}$ slice thickness and $0.4 \mathrm{~mm}$ interval at $70 \%, 75 \%$, and $80 \%$ of the R-R interval for coronary analysis and a second series at $1.25 \mathrm{~mm}$ slice thickness and $1.25 \mathrm{~mm}$ interval with 10 cardiac phases from $5 \%$ to $95 \%$ of the R-R interval for functional analysis. Additional coronary analysis phases were reconstructed as needed to address motion artifacts on the standard phases. Examinations were interpreted by a thoracic radiologist (GG) with 5 years experience in cardiac CT in conjunction with a cardiologist (ID). Interpretation and postprocessing were performed using an Advantage workstation (GEHC) or an iNtuition workstation (TeraRecon, Inc., San Mateo, CA).

Severe CAD was defined as luminal diameter narrowing estimated to be greater than $70 \%$ in at least one vessel or more than $50 \%$ left main coronary stenosis. Coronary stenosis that did not meet the defined criteria for severe was termed mild to moderate CAD. A diagnosis of chemotherapy-induced cardiomyopathy was established by LV dysfunction with prior cardiotoxic chemotherapy exposure and absence of significant obstructive CAD. Thrombocytopenia was defined as platelet count less than 50,000 per $\mu \mathrm{L}$, and leucopenia as white blood cell count below 4,000 per $\mu \mathrm{L}$. 

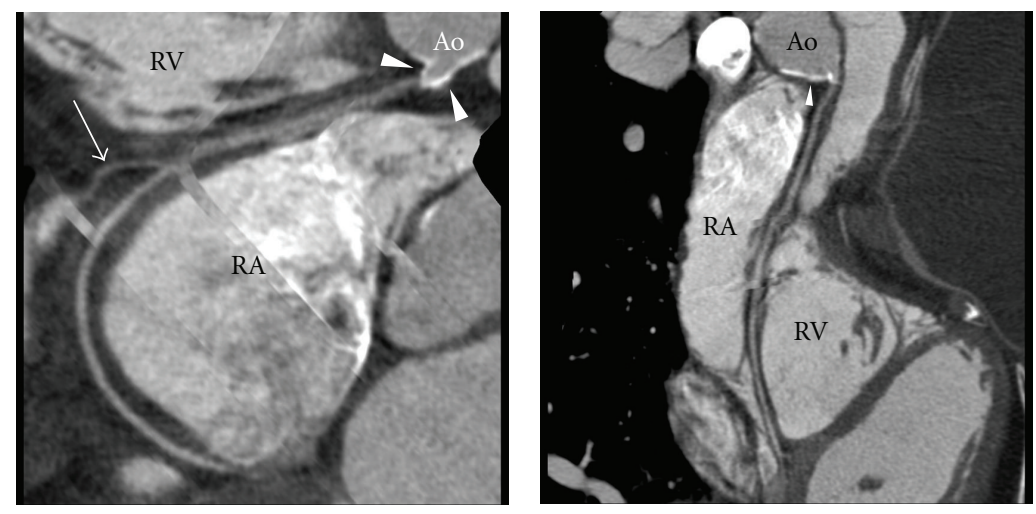

(a)

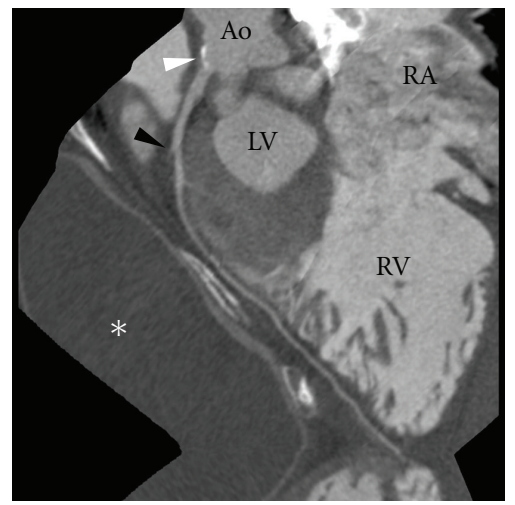

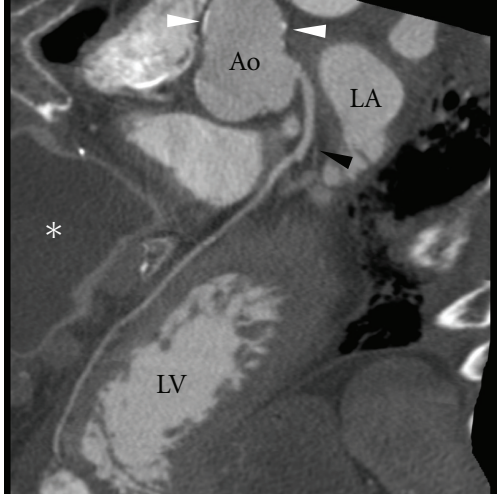

(b)

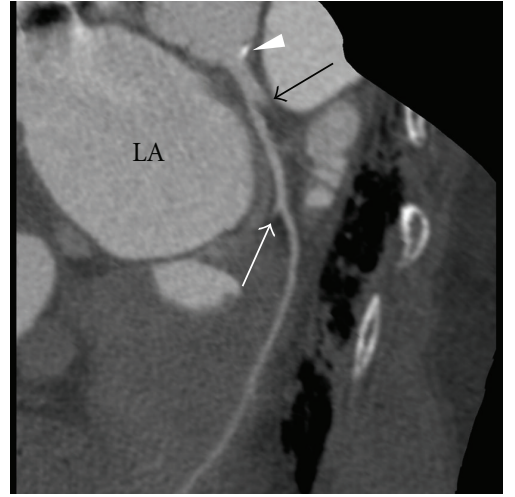

(c)

FIGURE 1: 48-year-old female with history of childhood lymphoma treated with mediastinal radiation therapy and anthracycline chemotherapy at age 12 , presented with fatigue and decreased left ventricular ejection fraction by echocardiogram. Coronary CT angiography shows nonobstructive coronary plaques and supports the diagnosis of chemotherapy-induced cardiomyopathy. (a) Curved reformat view of the right coronary artery (RCA) shows nonocclusive calcific ostial plaque (arrowheads) calcific plaque in the aortic root. The first acute marginal branch is identified (arrow). Also noted are misregistration artifacts. (b, c) Curved reformat views of the left anterior descending (LAD) (b) and circumflex (c) coronary arteries show calcific plaque (white arrowhead) in the aortic root at the origin of the left main coronary artery. The first diagonal artery (black arrowhead) is identified on the LAD view (b). The LAD origin (black arrow) and distal circumflex artery continuation (white arrow) are identified on the circumflex view (c). No significant narrowing is seen in any of the major coronary arteries. $\mathrm{LA}=$ left atrium. $\mathrm{LV}=$ left ventricle. $\mathrm{RA}=$ right atrium. $\mathrm{RV}=$ right ventricle. Ao $=$ Aortic root. $*=$ breast prosthesis.

Medical management of CAD was defined as treatment based on coronary calcification and/or the presence of plaque (which defines atherosclerotic disease and therefore identifies more aggressive secondary prevention targets).

CAD risk factor modification was defined as primary prevention step in absence of demonstrated CAD, which, for nondiabetic patients, has less stringent criteria.

\section{Results}

Eighty CCTAs were performed during the first year at our center. Baseline demographics, CAD risk factors and proportions of hematological malignancies are shown in Table 1.

The mean age of the patient population was $58.8 \pm 12.8$ years with $56 \%$ males. Hypertension was found in $48 \%$ of the cases, hyperlipidemia in $36 \%$ of cases, diabetes mellitus in $13 \%$ and established CAD in $9 \%$ of cases. About $45 \%$ of patients had hematological malignancy (leukemia and lymphoma) whereas the rest had a solid tumor.

The most common indication for obtaining a CCTA was LV dysfunction ( $41 \%$ of cases) followed by chest pain and or dyspnea in $40 \%$ of the cases (Table 2). About $20 \%$ CCTA was obtained for atypical symptoms with abnormal stress test and $10 \%$ were for atypical symptoms with abnormal biomarkers. Miscellaneous indications were present in 13 patients (16.3\%), including suspected cardiac tumor and/or thrombus or pulmonary embolism. Indications were not mutually exclusive and some patients had more than one at the time of CCTA.

Overall, 72 pts (90\%) had at least one relative contraindication to ICA (Table 3). The majority of the patients (61\%) who underwent a CCTA had a low to moderate pretest probability for CAD. Concomitant radiation or chemotherapy was present in $25 \%$ of cases and rest (19\%) had leucopenia and/or thrombocytopenia. 


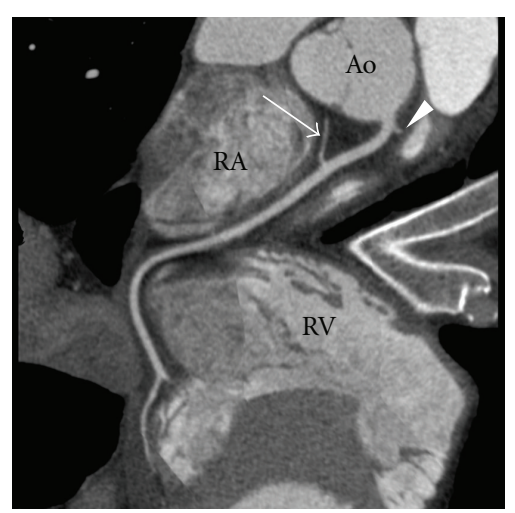

(a)

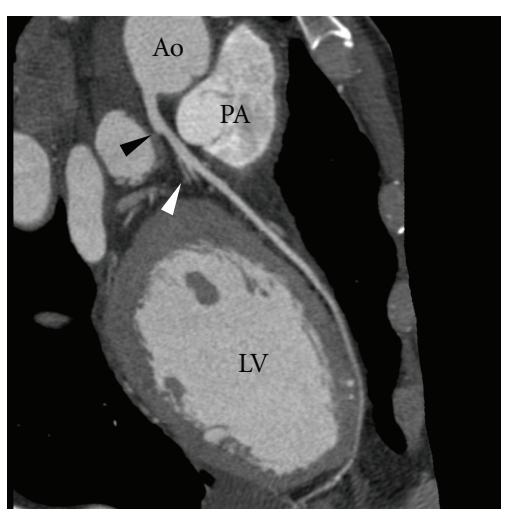

(b)

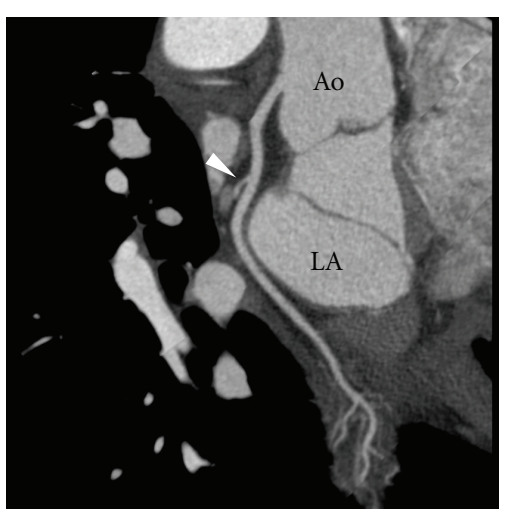

(c)

FIGURE 2: 53-year-old male with metastatic melanoma underwent testing prior to Interleukin-2 (IL-2) therapy. Resting electrocardiogram and echocardiogram are normal. Dobutamine stress echocardiogram shows inferior wall inducible ischemia. IL-2 is held while coronary CT angiography is performed. Based on CT findings, IL-2 is started and given uneventfully. (a) Curved reformat view of the right coronary artery shows no atherosclerotic plaque. The conus branch (arrowhead) and sinoatrial nodal branch (arrow) are identified. (b) Curved reformat view of the left anterior descending artery shows no atherosclerotic plaque. The circumflex artery origin (black arrowhead) and first diagonal artery (white arrowhead) are identified. (c) Curved reformat view of the circumflex coronary artery shows no atherosclerotic plaque. The first obtuse marginal branch (arrowhead) is identified. $\mathrm{LA}=$ left atrium. $\mathrm{LV}=$ left ventricle. $\mathrm{RA}=$ right atrium. $\mathrm{RV}=$ right ventricle. Ao $=$ Aortic root. $\mathrm{PA}=$ pulmonary artery root.

TABle 5: Cardiac treatment decisions based on cardiac computed tomographic angiography findings.

\begin{tabular}{lc}
\hline Cardiac treatment decisions & $N(\%)$ \\
\hline ICA $^{*}$ only & $8(10)$ \\
ICA followed by CABG & \\
Medical management of nonobstructive CAD & $\ddagger$ \\
CAD risk factors modification & $30(37.5)$ \\
$\begin{array}{l}\text { Management of chemotherapy-induced } \\
\text { cardiomyopathy }\end{array}$ & $20(25)$ \\
\hline
\end{tabular}

*ICA: invasive coronary angiography.

†CABG: coronary artery bypass grafting.

${ }^{\ddagger}$ CAD: coronary artery disease.

Coronary findings in CCTA are detailed in Table 4. Normal coronaries were found in $26.3 \%$ of patients, while mild to moderate CAD was found in $46 \%$ and severe CAD in $28 \%$.

Cardiac treatment decisions based on CCTA findings are outlined in Table 5.

Due to concomitant advanced cancer or patient refusal, only 12 out of 22 patients with severe CAD by CCTA underwent ICA, including 4 patients who eventually underwent coronary artery bypass grafting (CABG). The majority of the patients were managed medically, with $38 \%$ of the patients treated medically for nonobstructive CAD and $23 \%$ of patients treated for chemotherapy-induced cardiomyopathy (Figure 1). In 25\% of the cases the result of CCTA led to CAD risk factor modifications.

A total of 41 pts $(51.3 \%)$ had their cancer management altered based on CCTA findings (Table 6). In 24\% of cases, these findings directly influenced the decision to restarting chemotherapy (Figure 2), while in 9\% of cases, they led to
TABLE 6: Impact of cardiac computed tomographic angiography on cancer-related treatment decisions.

\begin{tabular}{lc}
\hline Cancer treatment decisions & $N(\%)$ \\
\hline Proceed with chemotherapy & $19(23.8)$ \\
Proceed with stem cell transplant evaluation & $8(10.0)$ \\
Proceed with cancer surgery & $3(3.8)$ \\
Proceed with radiation therapy & $2(2.5)$ \\
Change chemotherapy agents due to cardiomyopathy & $7(8.8)$ \\
Hold chemotherapy for invasive coronary angiography & $2(2.5)$ \\
\hline
\end{tabular}

a change in chemotherapy agents based on a diagnosis of chemotherapy-induced cardiomyopathy. In 2.5\% of cases, chemotherapy was temporarily discontinued in order to proceed with invasive angiography (Figure 3). In 10\% of cases, exclusion of significant CAD by CCTA allowed for stem cell transplant evaluation to proceed. In $4 \%$ of cases, cancer surgery was performed without further cardiac testing, and $2.5 \%$ of cases subsequently started radiation therapy.

\section{Discussion}

The present study shows that CCTA can have important consequences in evaluating cancer patients with suspected coronary disease, often leading to important decision in the care of both cancer and heart disease. For more than half the patients in our study, CCTA had a significant impact on their cancer care. Coexistence of CAD and malignancy often creates a therapeutic and diagnostic challenge: faced with two potentially life-threatening conditions, prioritization of care is paramount. This is because cancer, its treatment, and complications may render commonly used cardiac tests and 


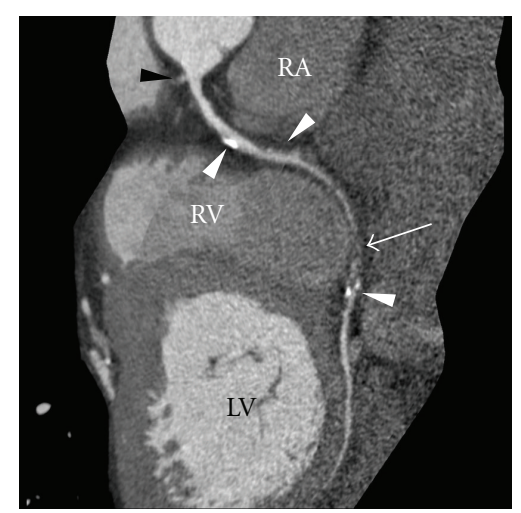

(a)

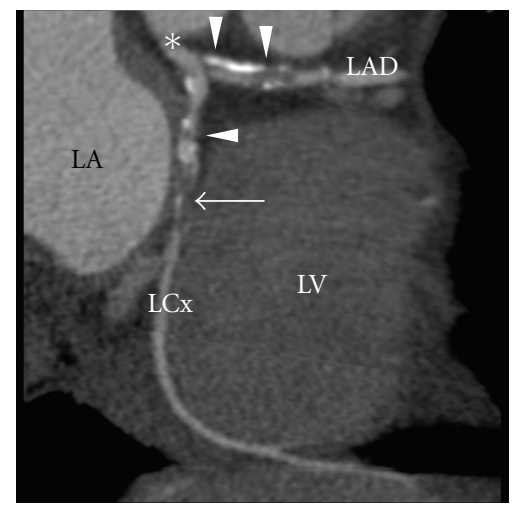

(c)

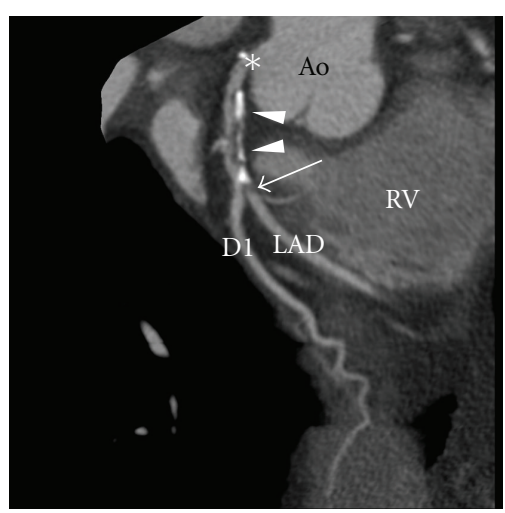

(b)

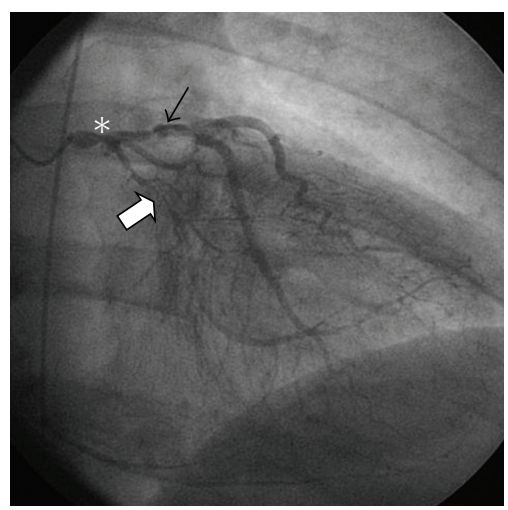

(d)

FIGURE 3: 61-year-old male with aggressive large B-cell lymphoma developed chest pain after the first cycle of chemotherapy. Electrocardiogram and cardiac enzyme measurements are consistent with NSTEMI. Echocardiography demonstrated a decrease in left ventricular ejection fraction from a normal baseline to $40 \%$. Due to postchemotherapy thrombocytopenia and leucopenia, an invasive coronary angiogram could not be obtained. Based on the coronary CT angiogram results, further chemotherapy was placed on hold. Invasive coronary angiography was performed after recovery of blood counts, followed by coronary artery bypass grafting. Two weeks later, the patient restarted chemotherapy. (a) Curved reformat view of the right coronary artery shows scattered calcific and noncalcified atherosclerotic plaque (white arrowheads) with possible occlusion (arrow) near the junction of the mid and distal segments. The conus branch origin (black arrowhead) is identified. (b) Curved reformat view of the left anterior descending artery (LAD) shows calcific and noncalcified atherosclerotic plaque (white arrowheads) in the proximal LAD, with focal near occlusion (arrow) of the LAD at the origin of the first diagonal (D1). Also noted is a mixed ostial left main artery plaque (asterisk). (c) Curved reformat view of the circumflex coronary artery (LCx) and proximal LAD shows calcific and noncalcified atherosclerotic plaque (arrowheads) in the proximal segments of both vessels. The LCx appears occluded at the junction of the proximal and middle segments (arrow). The ostial left main artery plaque in this projection appears noncalcified and significantly stenotic (asterisk). LA = left atrium. $\mathrm{LV}=$ left ventricle. $\mathrm{RA}=$ right atrium. $\mathrm{RV}=$ right ventricle. Ao = Aortic root. (d) Invasive angiogram demonstrating same findings with good correlation. The left main coronary is significantly stenosed (asterisk). The left anterior descending artery shows multiple high grade stenoses (thin arrow) and the left circumflex artery is occluded (thick arrow).

therapies difficult, if not contraindicated. Cancer patients receiving chemotherapy and experiencing bone marrow suppression are at higher risk of procedural complications should they undergo ICA routinely. In other instances, postoperative status, ongoing infections, or radiation therapy may also preclude an invasive approach. In fact, a large majority of our patients had at least one relative contraindication to ICA, and as such would have been less likely to be fully assessed without CCTA. This often leads to the use of noninvasive stress imaging, traditionally echocardiography and nuclear imaging for a functional assessment of the coronaries. However, neither technique is able to show nonobstructive CAD, nor are they free of false positives [8] (which could lead to unnecessary ICA [9]) and false negatives (which could fail to correctly identify cancer patients with significant CAD).

CCTA also allowed the accurate diagnosis of CIC, which has important implications in ongoing cancer care. Current guidelines for the diagnosis of CIC recommend a coronary angiogram to exclude the presence of significant CAD. The ACC/AHA guidelines for the diagnosis and management of heart failure updated in 2009 [2] recognize the use of noninvasive imaging for detection of ischemia in patients presenting with heart failure, if revascularization is contemplated, with the caveat that stress testing may not reliably distinguish between ischemic and nonischemic etiologies. 
This emphasizes the need for coronary anatomic assessment in these patients. In our practice in a tertiary cancer center, most patients with exposure to potentially cardiotoxic chemotherapy who experience a decrease in LV function are considered for angiography. Prior to the use of CCTA, however, many could not undergo ICA, and a conclusive diagnosis was often lacking. As we have shown, however, significant $\mathrm{CAD}$ in the setting of a cardiomyopathy could accurately be excluded, therefore confirming a suspicion of CIC in a quarter of our patients (Figure 1).

Noninvasive coronary artery imaging is therefore appealing in many instances in this patient population, particularly because of its high negative predictive value. Indeed, CCTA can conclusively demonstrate a normal coronary tree, therefore alleviating the need for ICA [10]. By accurately defining the coronary anatomy, CCTA in fact allowed the prioritization of cancer and cardiac care in our patients, in some cases, by excluding significant CAD suspected either based on cardiac biomarkers, symptoms, or previous testing (Figure 2). The advantage of adequate identification of underlying severe $\mathrm{CAD}$ in certain circumstances would allow stratification of patients who need modification of their chemotherapeutic regimen or who may benefit from concurrent treatment with cardioprotective therapeutic agents that promote positive remodeling, such as $\beta$-adrenergic blockers, angiotensin converting enzyme inhibitors, or angiotensin receptor blockers. In others, by identifying patients who needed their cancer care to be put on hold until more pressing cardiac issues could be addressed, CCTA also prevented undue delays in cancer care by seamlessly integrating with the flow of care.

Our results in a group of cancer patients with low to intermediate pretest probability for CAD on the basis of risk factors alone suggest that it may be more prevalent than suspected. Indeed, we found that nearly a third of pts had severe $\mathrm{CAD}$, and $5 \%$ of total population underwent CABG. This emphasizes the importance of coordinating cancer and cardiac care, as both can coexist with equal severity in the same patients. In fact, some cases will require the interruption of chemotherapy until the patient has undergone coronary revascularization (Figure 3). The addition of noninvasive coronary imaging therefore adds an invaluable tool in the approach to these difficult cases.

Our study describes a single center experience, and included patients referred for CCTA. In particular, there is no control group, and therefore no direct comparison to other noninvasive modalities (stress testing) can be drawn. Whether CCTA as a first step would compare favorably to other noninvasive modalities needs further study, particularly as a cost and radiation reduction measure. Although we reported short-term outcomes in terms of cancer and cardiac care, long-term followup will be needed to show definitive improvement in overall outcomes.

\section{Conclusions}

Cardiac computed tomographic angiography is an attractive imaging modality in cancer patients with suspected structural heart disease when invasive coronary angiography may be too risky or impractical and allows improved coordination of cancer and cardiac care.
Abbreviations
CABG: coronary artery bypass grafting
CIC: chemotherapy-induced cardiomyopathy
CCTA: cardiac computed tomographic angiography
CAD: coronary artery disease
ECG: electrocardiogram
ICA: invasive coronary angiography
LV: left ventricular.

\section{Conflicts of Interest}

The authors have no conflicts of interest to disclose.

\section{References}

[1] E. T. Yeh, A. T. Tong, D. J. Lenihan et al., "Cardiovascular complications of cancer therapy: diagnosis, pathogenesis, and management," Circulation, vol. 109, no. 25, pp. 3122-3131, 2004.

[2] S. A. Hunt, W. T. Abraham, M. H. Chin et al., "2009 focused update incorporated into the ACC/AHA 2005 guidelines for the diagnosis and management of heart failure in adults: a report of the American College of Cardiology Foundation/American Heart Association Task Force on Practice Guidelines," Journal of the American College of Cardiology, vol. 53, no. 15, pp. e1-e90, 2009.

[3] M. R. Patel, G. J. Dehmer, J. W. Hirshfeld, P. K. Smith, and J. A. Spertus, "ACCF/SCAI/STS/AATS/AHA/ASNC 2009 Appropriateness Criteria for Coronary Revascularization. A Report by the American College of Cardiology Foundation Appropriateness Criteria Task Force, Society for Cardiovascular Angiography and Interventions, Society of Thoracic Surgeons, American Association for Thoracic Surgery, American Heart Association, and the American Society of Nuclear," Journal of the American College of Cardiology, vol. 53, no. 6, pp. 530-553, 2009.

[4] P. K. Vanhoenacker, M. H. Heijenbrok-Kal, R. Van Heste et al., "Diagnostic performance of multidetector CT angiography for assessment of coronary artery disease: meta-analysis," Radiology, vol. 244, no. 2, pp. 419-428, 2007.

[5] G. L. Raff, M. J. Gallagher, W. W. O’Neill, and J. A. Goldstein, "Diagnostic accuracy of noninvasive coronary angiography using 64-slice spiral computed tomography," Journal of the American College of Cardiology, vol. 46, no. 3, pp. 552-557, 2005.

[6] A. W. Leber, A. Knez, F. Von Ziegler et al., "Quantification of obstructive and nonobstructive coronary lesions by 64 -slice computed tomography: a comparative study with quantitative coronary angiography and intravascular ultrasound," Journal of the American College of Cardiology, vol. 46, no. 1, pp. 147154, 2005.

[7] G. Mowatt, E. Cummins, N. Waugh et al., "Systematic review of the clinical effectiveness and cost-effectiveness of 64-slice or higher computed tomography angiography as an alternative to invasive coronary angiography in the investigation of coronary artery disease," Health Technology Assessment, vol. 12, no. 17, 2008 . 
[8] S. C. Danciu, C. J. Herrera, P. J. Stecy, E. Carell, F. Saltiel, and J. L. Hines, "Usefulness of multislice computed tomographic coronary angiography to identify patients with abnormal myocardial perfusion stress in whom diagnostic catheterization may be safely avoided," American Journal of Cardiology, vol. 100, no. 11, pp. 1605-1608, 2007.

[9] B. J. W. Chow, A. Abraham, G. A. Wells et al., "Diagnostic accuracy and impact of computed tomographic coronary angiography on utilization of invasive coronary angiography," Circulation: Cardiovascular Imaging, vol. 2, no. 1, pp. 16-23, 2009.

[10] T. Schlosser, O. K. Mohrs, A. Magedanz et al., "Noninvasive coronary angiography using 64-detector-row computed tomography in patients with a low to moderate pretest probability of significant coronary artery disease," Acta Radiologica, vol. 48 , no. 3 , pp. 300-307, 2007. 


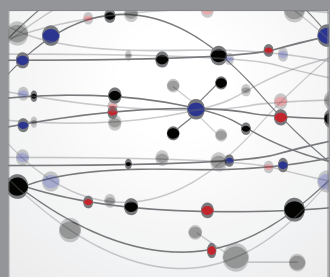

The Scientific World Journal
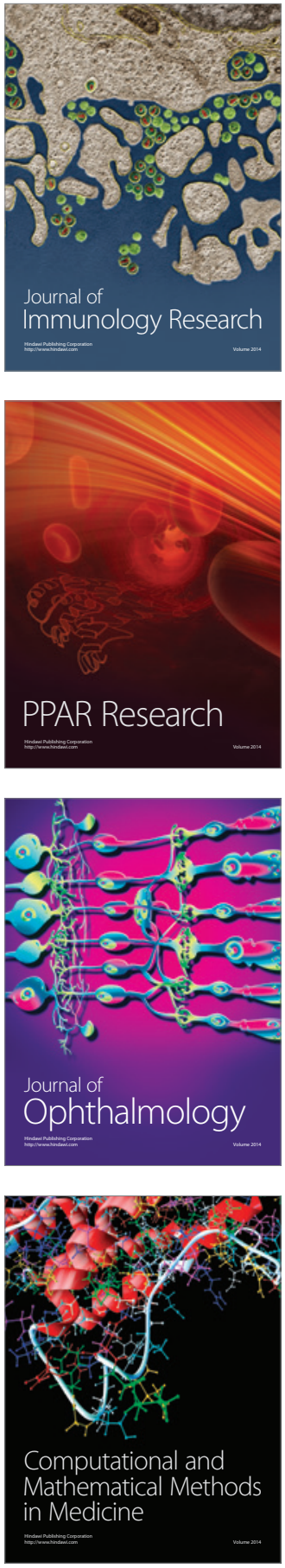

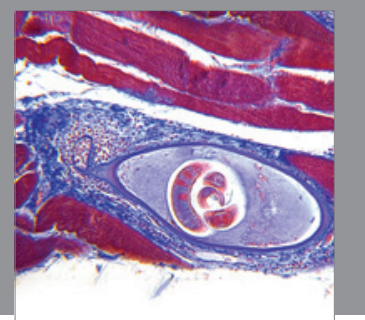

Gastroenterology

Research and Practice
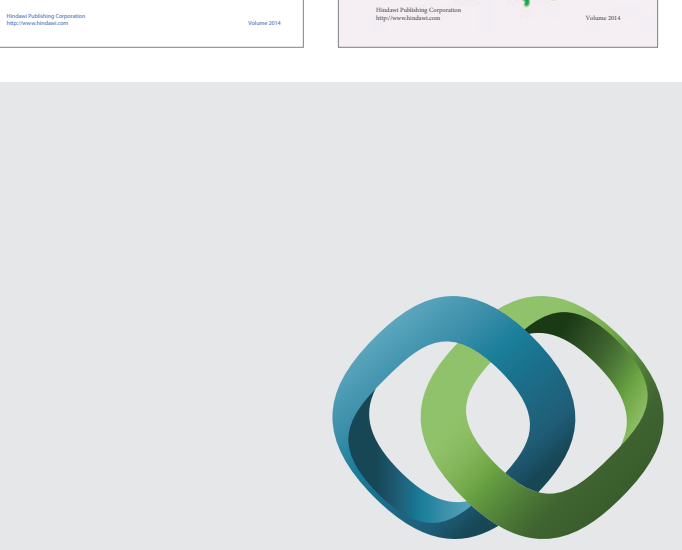

\section{Hindawi}

Submit your manuscripts at

http://www.hindawi.com
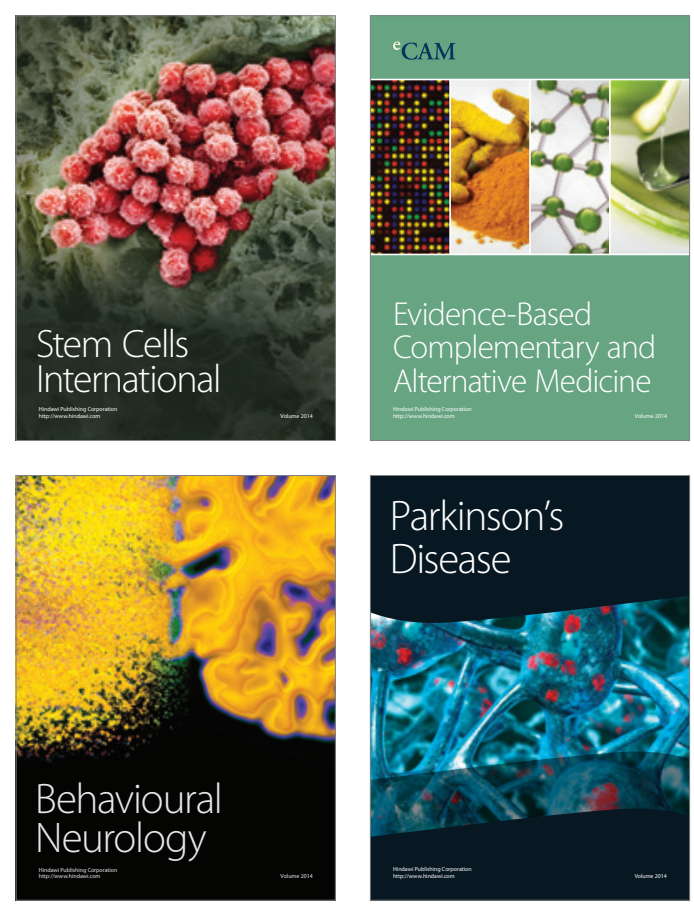

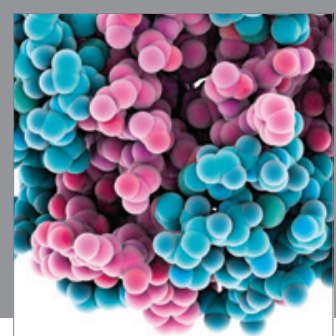

Journal of
Diabetes Research

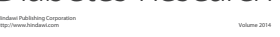

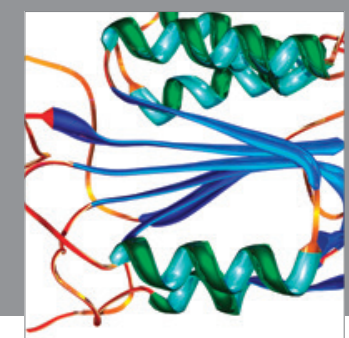

Disease Markers
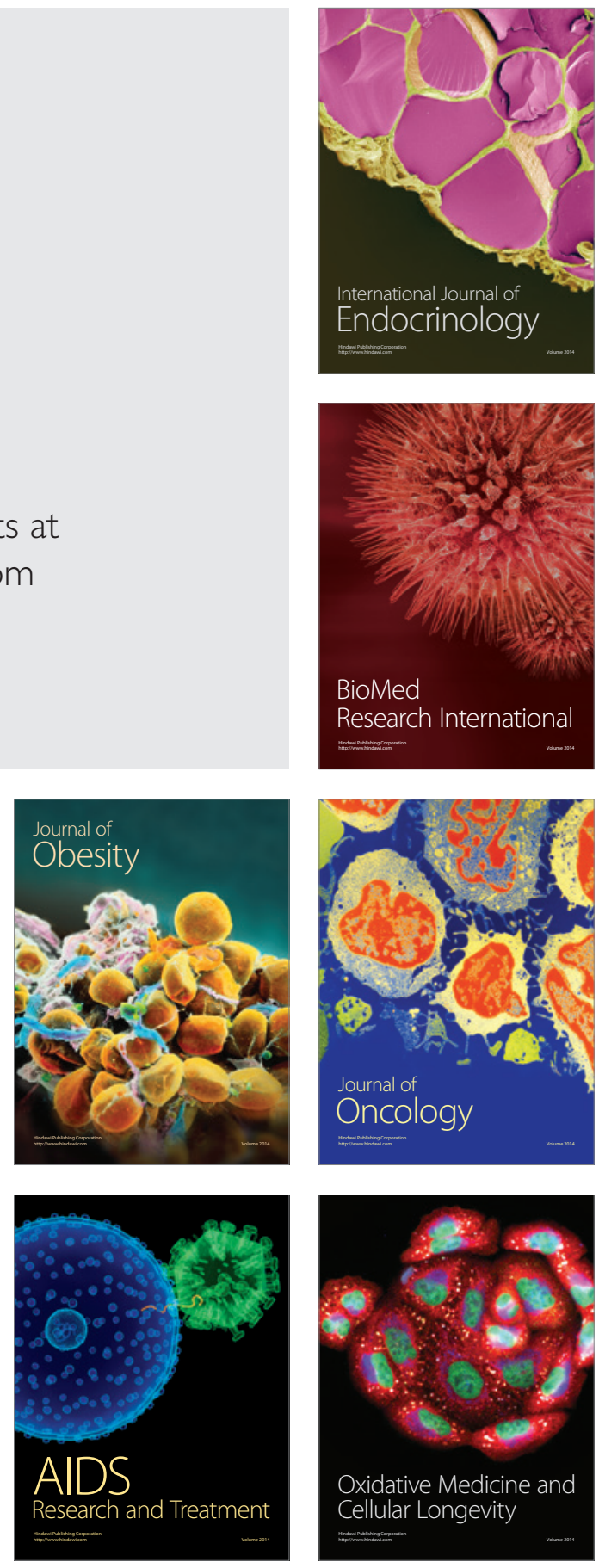